\title{
Perfil antropométrico e fisiológico de atletas de futsal da categoria sub-20 e adulta
}

\author{
Anthropometrical and physiological profile of futsal players of the \\ under-20 and adults categories
}

\author{
F.A. Barbieri, R.A. Barbieri, M.R. Queiroga, W.C. Santana, E. Kokubun
}

ARTIGO ORIGINAL | ORIGINAL ARTICLE

\begin{abstract}
RESUMO
O objetivo do estudo foi determinar o perfil antropométrico, bem como as diferentes capacidades fisiológicas, de atletas de futsal do sexo masculino e comparar entre as categorias Sub-20 e adulta. Participaram do estudo 12 atletas pertencentes a uma equipe da categoria Sub-20 e 12 atletas pertencentes a uma equipe da categoria adulta. Os participantes foram submetidos a uma rotina de avaliação que envolvia medidas antropométricas (massa corporal, estatura, espessura das dobras cutâneas, perímetro corporais e diâmetros ósseos) e testes para estimativa da capacidade aeróbia e anaeróbia. Os resultados não indicaram diferenças em relação ao perfil antropométrico e fisiológico entre as categorias Sub-20 e adulta. Concluiu-se que as categorias Sub-20 e adulta apresentam similar perfil antropométrico e desempenho aeróbio e anaeróbio, sugerindo que relacionado aos aspetos estudados é recomendada a promoção dos atletas da categoria Sub-20 para a categoria adulta. No entanto, outros indicadores não estudados, como os aspetos técnicos, táticos e psicológicos, podem ser importantes para auxiliar na compreensão desta fase de transição e no treinamento dos atletas.

Palavras-chave: avaliação, somatotipo, capacidade aeróbia e anaeróbia, futsal
\end{abstract}

ABSTRACT

The aim of this study was to determine the anthropometrical and physiological profile of futsal players and compare between under-20 and adults categories. Twenty four male athletes futsal participated in the study. They played in two teams of futsal-level state: a team with players under-20 (12 athletes); and another with players of the adults category (12 athletes). The participants performed a routine assessment involving anthropometric measurements (body mass, size, thickness of skin folds, perimeter body and bone diameter) and tests to estimate the aerobic and anaerobic capacity. The results did not indicate differences in relation to the anthropometrical and physiological profile between the under-20 and adults categories. We concluded that the promotion of athletes from the under- 20 category for the adult's category taking into account the anthropometrical profile and aerobic and anaerobic performance indicators is recommended. However, other indicators, such as the technical, tactical and psychological aspects are important to help in understanding this promotion phase and in the training.

Keywords: assessment, somatotype, aerobic and anaerobic capacity, futsal

Submetido: 03.10.2011 | Aceite: 07.12.2012

Fabio Augusto Barbieri, Ricardo Augusto Barbieri e Eduardo Kokubun. Universidade Estadual Paulista, Departamento de Educação Física, Rio Claro, São Paulo, SP, Brasil.

Marcos Roberto Queiroga. Universidade Estadual do Centro Oeste, UNICENTRO, Guarapuava, Paraná, PR, Brasil. Wilton Carlos Santana. Universidade Estadual de Londrina, Departamento de Esportes, UEL, Londrina, Paraná, PR, Brasil.

Endereço para correspondência: Prof. Fabio Augusto Barbieri, Rua 8b, 1171 - Bela Vista, CEP: 13506-739 - Rio Claro - São Paulo, SP, Brasil.

E-mail: barbieri@rc.unesp.br 
O futsal como tantas outras modalidades coletivas requer intensa participação do metabolismo aeróbio e anaeróbio (Barbero-Alvarez, Soto, Barbero-Alvarez, \& Granda-Vera, 2008; Castagna, Belardinelli, Impellizzeri, Abt, Coutts, \& D'Ottavio, 2007; Dogramaci \& Watsford, 2006; Lima, Silva, \& Souza, 2005). Frequentemente são exigidos esforços intermitentes de curta duração e de alta intensidade apoiados em grande quantidade de ações realizadas com e sem bola (Barbieri, Benites, \& Machado, 2007). Entretanto, devido às características do futsal (substituições ilimitadas, tempo de jogo cronometrado, tamanho da quadra, entre outras) verifica-se uma relação particular entre os metabolismos (relação esforço-pausa), o que justifica uma preparação física única e específica para a modalidade.

Estudos têm quantificado aspetos antropométricos e fisiológicos de atletas de futsal e fornecido subsídios importantes para o planejamento do treinamento. Comparações entre as categorias são importantes para caracterizar as diferenças e as similaridades nos aspetos antropométricos (caracterizado pelas medidas antropométricas e somatotipo) e fisiológicos (caracterizado pelo desempenho aeróbio e anaeróbio). Assim, o treinamento e as transições entre as categorias tornam-se mais especializados e menos desgastantes para os atletas. No entanto, embora estudos tenham sugerido diferenças entre as categorias em formação nos aspetos antropométricos e fisiológicos (Dias, Carvalho, Souza, Avelar, Altimari \& Cyrino, 2007; Avelar et al., 2008; Ré, Teixeira, Massa, \& Böhme, 2003; Cyrino, Altimari, Okano, \& Coelho, 2002), não há relato de comparações realizadas entre a categoria Sub-20 (última categoria antes de acender para a categoria adulta - atletas com idade entre 18 e 20 anos) e a adulta.

Neste sentido, quando comparamos, analisando resultados apresentados por diferentes estudos (Alvarez, D’Ottavio, Vera \& Castagna, 2009; Avelar et al., 2008; Leal Junior, Souza,
Magini \& Martins, 2006; Lima et al., 2005), as características antropométricas da categoria Sub-20 e adulta, nota-se similaridade entre as categorias (categoria adulta: massa corporal entre 68 e $76 \mathrm{~kg}$, estatura entre 1.73 e 1.80 $\mathrm{m}$, índice de massa corporal em torno de 24 $\mathrm{kg} / \mathrm{m}^{2}$, percentual de gordura entre 9 e $10 \%$ e perfil com predominância da mesomorfia; categoria Sub-20: média de massa corporal de $68.5 \mathrm{~kg}$, de estatura de $1.77 \mathrm{~m}$ e de índice de massa corporal de $21.7 \mathrm{~kg} / \mathrm{m}^{2}$ ). Ainda, como as regras básicas do jogo de futsal são similares entre as categorias e entre os sexos, supõe-se que a mesomorfia seja o somatotipo mais indicado para a modalidade (Queiroga, Ferreira \& Romanzini, 2005; Queiroga, Ferreira, Pereira $\&$ Kokubun, 2008). Com relação às características fisiológicas, é conhecido que na categoria adulta de futsal masculino a intensidade do deslocamento é alterada a cada $3.28 \mathrm{~s}$, sendo que $26 \%$ destes ocorrem em alta intensidade. Ainda, são exigidos em torno de 13 a 39 corridas em alta intensidade a cada $79 \mathrm{~s}$ e consumo de oxigênio $\left(\mathrm{VO}_{2 \text { máx }}\right)$ entre 40.1 a $57.1 \mathrm{ml} \cdot \mathrm{kg}^{-1} \cdot \mathrm{min}^{-1}$ (Castagna, D'Ottavio, Vera, \& Barbero Alvarez, 2009; Avelar et al., 2008; Dogramaci \& Watsford, 2006). Para as categorias de formação no futsal, apenas um estudo analisou o desempenho aeróbio e anaeróbio de uma categoria de formação (Sub-17), revelando valores de $84 \%$ e $75 \%$ da frequência cardíaca máxima e do $\mathrm{VO}_{2 \text { máx }}$, respetivamente, e que $9 \%$ do jogo foi executado em intensidade maior que $70 \%$ da frequência cardíaca máxima (Castagna et al., 2007). Com isso, parece que as exigências fisiológicas entre as categorias são distintas, sendo que as exigências para a categoria adulta são maiores.

Entretanto, comparações destas características entre as equipes Sub-20 e adultas seriam interessantes para a promoção de atletas para a categoria adulta. Devido a uma série de situações inusitadas, como convivência com atletas mais velhos, estilo de jogo, exigências e cobranças, há indicação de que a transição 
entre a categoria Sub-20 e a adulta é a mais desconfortável para os atletas (Ré, Teixeira, Massa, \& Böhme, 2003). Estas novas situações podem acarretar queda de rendimento esportivo, overtraining e desmotivação, que muitas vezes não são considerados/identificadas na prática. Como as normas da CBFS permite a participação de atletas da categoria Sub-20 nas equipes da categoria adulta, o questionamento sobre o momento correto de ingressar o atleta na categoria adulta é evidenciado. Desta forma, compreender aspetos relacionados a antropometria predominante e ao desempenho fisiológico relacionados as duas categorias podem auxiliar neste processo de transição e também permitir ajustes precisos nas cargas de treinamento com a finalidade de maximizar o desempenho sem sobrecarregar os atletas.

Sendo assim, o objetivo do estudo foi determinar o perfil antropométrico, bem como diferentes capacidades fisiológicas, tendo por base a caracterização da capacidade aeróbia e anaeróbia de atletas de futsal do sexo masculino e comparar entre as categorias Sub-20 e adulta. A hipótese do estudo é que os atletas da categoria adulta apresentem parâmetros aeróbios e anaeróbios melhores do que os atletas da categoria Sub-20, porém perfil antropométrico similar entre as categorias.

\section{MÉTODO}

Participaram do estudo 24 atletas do sexo masculino pertencentes a duas equipes de futsal, sendo que uma das equipes era composta por jogadores da categoria Sub-20 (12 atletas) e outra por jogadores da categoria adulta (12 atletas). Ambas as equipes disputam campeonatos de nível estadual (estado de São Paulo Brasil) da Segunda divisão e torneios regionais da respetiva categoria.

Todos os atletas não apresentavam histórico de problemas de saúde e assinaram um termo de consentimento livre e esclarecido aprovado pelo comitê de ética local. Os atletas de cada categoria realizavam treinamento quatro vezes por semana, de duas a três horas por dia, no período noturno. O treinamento das duas equipes consistia em estímulos físicos, técnicos e táticos (com e sem bola). Além disso, as equipes participavam de jogos duas vezes por semana em dias diferentes dos treinos. As duas equipes estavam em período de competição e por isso em fase similar de preparação durante as avaliações.

Os participantes foram submetidos a uma rotina de avaliação que envolvia medidas antropométricas (massa corporal, estatura, espessura das dobras cutâneas, perímetro corporais e diâmetros ósseos) e testes para estimativa da capacidade aeróbia e anaeróbia. Os testes e medidas foram realizados em dois dias com intervalo de $48 \mathrm{~h}$. No primeiro dia foram avaliadas as medidas antropométricas e a capacidade aeróbia, enquanto no segundo dia a capacidade anaeróbia.

\section{Medidas antropométricas}

A massa corporal foi mensurada por meio de uma balança antropométrica com precisão de $100 \mathrm{~g}$ e a estatura a partir de um estadiômetro de madeira com precisão de $0.1 \mathrm{~cm}$. As espessuras das dobras cutâneas foram mensuradas em milímetro com auxilio de um compasso Cescorf $^{\circledast}$. Foram destacadas as dobras nas regiões tricipital, subescapular, suprailíaca (supraespinhal), coxa e perna medial. Os diâmetros ósseos biepicôndiliano do úmero e do fêmur foram determinados por um paquímetro de metal $\left(\right.$ Somet $^{\circledR}$ ) com escala de medida de $0.1 \mathrm{~cm}$. As medidas de circunferência do braço contraído e da panturrilha foram realizadas com auxílio de uma fita métrica inextensível (Mabis $\left.{ }^{\circledR} J a p a n\right)$.

As medidas antropométricas permitiram o cálculo do índice de massa corporal, da densidade corporal, a partir do emprego do modelo de regressão proposto por Jackson, Pollock, \& Ward (1980) enquanto o percentual de gordura foi determinado de acordo com a sugestão de Siri (1961). O somatotipo corporal foi estimado 
seguindo as orientações de Heath e Carter (1967). As medidas antropométricas para o cálculo do somatotipo foram realizadas a partir das sugestões de Ross e Marfell-Jones (1982).

\section{Avaliação do componente aeróbio}

Para avaliar a capacidade aeróbia empregou-se o teste de corrida de vai e vem (Léger, Mercier, Gadoury, \& Lambertz, 1988). O teste consiste em uma série de corridas intermitentes e progressivas de $20 \mathrm{~m}$, iniciando com velocidade de $8 \mathrm{~km} / \mathrm{h}$ com incremento de 0.5 $\mathrm{km} / \mathrm{h}$ a cada minuto. O teste foi aplicado em uma quadra poliesportiva coberta, devidamente marcada e equipada. A capacidade aeróbia máxima foi estimada a partir da equação de regressão abaixo.

$$
\mathrm{VO}_{2 \max }=(6 \mathrm{x} \text { velocidade do teste })-24.4
$$

Esse protocolo demonstrou validade concorrente aceitável em adultos jovens brasileiros (homens, $r=.73$; mulheres, $r=.75$ ) (Duarte \& Duarte, 2001).

\section{Avaliação do componente anaeróbio}

Para avaliar a potência anaeróbia e o índice de fadiga foi utilizado o Running-based Anaerobic sprint test (RAST) (Zacharogiannis, Paradisis, \& Tziortzis, 2004). O teste consiste em seis corridas de $35 \mathrm{~m}$ em velocidade máxima com intervalos de $10 \mathrm{~s}$ e foi aplicado em uma pista de atletismo devidamente marcada e equipada. A potência anaeróbia de cada corrida e o índice de fadiga (IF) foram calculados por meio das equações abaixo.

$$
\begin{aligned}
& \text { Potência }=\frac{(\text { Peso } x \text { Estatura })^{2}}{\text { Tempo da corrida }} \\
& \text { I F }=\frac{(\text { Potência Máxima }- \text { Potência Mínima })}{\text { Tempo Total Corridas }}
\end{aligned}
$$

A partir da potência anaeróbia de cada corrida foi selecionada a potência máxima e calculada a potência média para cada partici- pante.

\section{Análise estatística}

Idade, massa corporal, estatura, índice de massa corporal, percentual de gordura, tempo de prática, somatotipo, $\mathrm{VO}_{2 \max }$, duração de cada corrida em velocidade máxima, potência anaeróbia máxima e média e índice de fadiga foram as variáveis utilizadas para comparar os grupos. A análise estatística foi realizada usando o pacote estatístico SPSS versão 15.0 para Windows ${ }^{\odot}$ (SPSS, Inc., Chicago, IL) com nível de significância de $p<.05$. Os dados foram agrupados e descritos em valores de média e desvio padrão. O teste de Shapiro Wilk foi utilizado para verificar a normalidade na distribuição dos dados. Em seguida, os grupos, categoria Sub-20 e adulta, foram comparados por meio do teste $t$ de Student para amostras independentes.

\section{RESULTADOS}

Características antropométricas

A análise estatística revelou diferença significativa somente para a idade $(t 22=-7.21, p$ $>$.001), obviamente favorecendo os atletas da categoria adulta. $\mathrm{O}$ somatotipo predominante apresentado pelos atletas da categoria Sub-20 e adulta foi o endo mesomorfo. Por sua vez, não diferiram significativamente entre os componentes de antropométricos (Tabela 1).

\section{Características fisiológicas}

Os atletas das diferentes categorias apresentaram similar $\mathrm{VO}_{2 \max }$, duração de cada corrida, potência anaeróbia máxima e média e para índice de fadiga (Tabela 2). Desta forma, os atletas da categoria Sub-20 e adulta apresentaram semelhante perfil fisiológico, considerando a capacidade aeróbia e anaeróbia. 
Tabela 1.

Características gerais e antropométricas dos atletas de futsal da categoria adulta e Sub-20. IMC - índice de massa corporal.

\begin{tabular}{ccc}
\hline & Sub-20 & Adulta \\
\hline Idade (anos) & $18.5 \pm 0.7^{*}$ & $22.4 \pm 1.5$ \\
Tempo de treino (meses) & $84 \pm 37$ & $113 \pm 64$ \\
Massa corporal (kg) & $70.3 \pm 8.8$ & $75.8 \pm 8.7$ \\
Estatura (cm) & $175.4 \pm 4.9$ & $175.8 \pm 7.9$ \\
Índice de massa corporal & $22.8 \pm 2.1$ & $24.5 \pm 2.2$ \\
(kg/m $\left.{ }^{2}\right)$ & $14.1 \pm 4.9$ & $14.5 \pm 3.6$ \\
Gordura relativa (\%) & $60.2 \pm 6.3$ & $64.7 \pm 6.6$ \\
Massa magra (kg) & $3.6 \pm 1.5$ & $3.8 \pm 1.1$ \\
Endomorfia & $4.2 \pm 0.7$ & $4.9 \pm 1.3$ \\
Mesomorfia & $2.3 \pm 0.7$ & $1.7 \pm 0.8$ \\
Ectomorfia & Endo & Endo \\
Somatotipo médio & mesomorfo & mesomorfo \\
\hline * categoria Sub-20 $\neq$ categora adulta, & . &
\end{tabular}

* categoria Sub-20 $\neq$ categoria adulta, $p>.001$.

\section{DISCUSSÃo}

As hipóteses do estudo foram confirmadas parcialmente uma vez que os atletas da categoria Sub-20 e da adulta não diferiram em relação aos indicadores fisiológicos (desempenho aeróbio e anaeróbio). Por sua vez a similaridade entre as categorias em relação ao perfil antropométrico foi prevista inicialmente.

A semelhança do tempo de treinamento entre as categorias, que normalmente costuma favorecer a categoria adulta, proporciona um lastro de aptidão equiparado entre os atletas (Machado \& Gomes, 1999). Estudos mostram que atletas com tempo de treinamento próximos, sendo este realizado em intensidade e volume semelhante, apresentam desempenhos parecidos (Santana, França, Reis, \& Ribeiro, 2007). É possível que se o grupo de atletas da categoria adulta fosse mais velho e consequentemente com maior tempo de treinamento, aparecessem diferenças significantes, favorecendo a equipe da categoria adulta.

Desta forma, apesar dos atletas da categoria adulta $(22.4 \pm 1.6$ anos $)$ ser significativamente mais velhos do que os atletas da categoria Sub-20 (18.6 \pm .8 anos), a distância absoluta, isto é, aproximadamente quatro anos,
Tabela 2.

Características fisiológicas dos atletas de futsal da categoria adulta e Sub-20.

\begin{tabular}{|c|c|c|}
\hline & Sub-20 & Adulta \\
\hline \multirow[t]{3}{*}{$\begin{array}{c}\mathrm{VO}_{2 \operatorname{máx}} \\
\text { (ml.kg.min) }\end{array}$} & $50.3 \pm 4.6$ & $50.1 \pm 4.4$ \\
\hline & $5.4 \pm 0.3$ & $5.4 \pm 0.2$ \\
\hline & $5.4 \pm 0.5$ & $5.6 \pm 0.3$ \\
\hline \multirow{4}{*}{$\begin{array}{c}\text { Duração de cada } \\
\text { corrida (s) }\end{array}$} & $5.6 \pm 0.6$ & $5.9 \pm 0.2$ \\
\hline & $5.7 \pm 0.6$ & $5.9 \pm 0.2$ \\
\hline & $5.9 \pm 0.6$ & $6.1 \pm 0.4$ \\
\hline & $6.1 \pm 0.6$ & $6.2 \pm 0.4$ \\
\hline $\begin{array}{l}\text { Potência anaeróbia } \\
\text { máxima (watts) }\end{array}$ & $619.2 \pm 228.1$ & $588.8 \pm 109.5$ \\
\hline $\begin{array}{l}\text { Potência anaeróbia } \\
\text { média (watts) }\end{array}$ & $499.2 \pm 202.6$ & $468.3 \pm 66.2$ \\
\hline $\begin{array}{l}\text { Índice de fadiga } \\
\text { (watts/s) }\end{array}$ & $6.8 \pm 3.5$ & $6.5 \pm 2.7$ \\
\hline
\end{tabular}

é pequena. Nesta fase de desenvolvimento não há nenhuma transformação orgânica e fisiológica importante que destaque o metabolismo energético em indivíduos com idades entre 18 e 22 anos (Weineck, 2005), o que aumenta a relevância do treinamento para efetivar alterações neste aspeto. É interessante ressaltar que a idade da categoria adulta deste estudo é discretamente menor a de outras equipes profissionais (25 a 26 anos) (Avelar et al., 2008; Barbero-Alvarez et al., 2008; Zacharogiannis et al., 2004).

A preparação geral no futsal adota a categoria adulta como base para os treinamentos da categoria Sub-20 (Arena \& Böhme, 2004; Rezer \& Shigunov, 2004). Semelhantes intensidades e volumes de treino são utilizados para estas categorias, o que proporciona desenvolvimento e desempenho similar entre as categorias. Juntamente com isso, existe a participação dos atletas da categoria Sub-20 nos jogos da categoria adulta e algumas vezes nos treinamentos. Esta prática comum contribui para que atletas Sub-20 desenvolvam elevada capacidade aeróbia e anaeróbia (Lima et al., 2005), equiparando-os aos atletas da categoria adulta. Do ponto de vista fisiológico, isto permitiria que 
atletas da categoria Sub-20 atuassem junto com a categoria adulta sem déficits no desempenho.

Com isso, a análise dos atletas, com relação aos parâmetros antropométricos e fisiológicos, pode caracterizar de forma geral a categoria adulta (Tabela 3), uma vez que diferenças entre as categorias não foram evidenciadas e muitos jogadores da categoria Sub-20 também fazem parte do plantel da equipe adulta. Assim, os resultados apresentados pelo presente estudo são similares ao encontrado por estudos anteriores que analisaram a categoria adulta de futsal (Alvarez et al., 2009; Castagna et al., 2009; Avelar et al., 2008; Dogramaci \& Watsford, 2006; Leal Junior et al., 2006), principalmente com relação a massa corporal, estatura, índice de massa corporal e somatotipo predominante. Entretanto, foi encontrado valores superiores com relação à percentual de gordura e inferiores para $\mathrm{VO}_{2 \text { máx }}$. Possivelmente a diferença no nível de competição disputada pelos atletas deste estudo e dos outros estudos, os diferentes métodos de treinamento e as limitações metodológicas de cada estudo, sejam as explicações para as diferenças encontradas nestes dois parâmetros.

Apesar da semelhança entre as categorias, a proficiência no jogo e a experiência no futsal poderiam dar mais condições ao atleta da categoria adulta aproveitar eficazmente os recursos físicos, técnicos, táticos e psicológicos (Amaral \& Garganta, 2005; Ré et al., 2003). Logo, para promover o atleta da categoria Sub-20 para a adulta, além de considerar o perfil antropométrico e fisiológico é importante analisar outros aspetos envolvidos com o contexto do futsal, como os fatores técnicos, táticos e psicológicos. A capacidade técnica do atleta é relevante para a proficiência e promoção para a categoria subsequente (Barbieri \& Gobbi, 2009). Como o futsal é um esporte de técnica aberta e, sobretudo um "jogo de informação" (Amaral \& Garganta, 2005), os atletas necessitam decodificar as informações situacionais (Corrêa \& Tani, 2006) para selecionar a ação mais adequada para determinado momento do jogo (Amaral \& Garganta, 2005; Garganta, 2002). Dessa maneira, mesmo apresentando elevada capacidade aeróbia e anaeróbia e somatotipo preferencial para o esporte em questão, os atletas da categoria Sub-20 podem ter dificuldades em selecionar a ação mais adequada. Por isso, muitas vezes atletas com alto nível técnico conseguem compensar seus baixos níveis fisiológicos durante a partida, principalmente atletas com mais experiência (Couto Junior, Moreno, Souza, Prado \& Machado, 2007). Além disso, a tática imposta pela equipe durante a partida pode ser decisiva (Duarte, 2008). Atletas mais proficientes no futsal aproveitam melhor os aspetos ofensivos e defensivos que ocorrem durante a prática de futsal, indicando a importância do aspeto tático para determinar a promoção do atleta para a equipe principal. Ainda, muitos atletas da categoria Sub-20 não estão preparados psicologica-

Tabela 3.

Média e desvio padrão das características gerais, antropométricas e fisiológicas da categoria adulta no futsal IF - índice de fadiga.

\begin{tabular}{cccccc}
\hline \multicolumn{2}{c}{ Características gerais e antropométricas } & & \multicolumn{2}{c}{$\begin{array}{c}\text { Características } \\
\text { Aeróbias e anaeróbias }\end{array}$} \\
\hline $\begin{array}{c}\text { Idade (anos) } \\
\begin{array}{c}\text { Tempo de treino } \\
\text { (meses) }\end{array}\end{array}$ & $20.4 \pm 2.2$ & $\begin{array}{c}\text { Percentagem } \\
\text { de gordura }\end{array}$ & $14.3 \pm 4.2$ & $\begin{array}{c}\text { VO }_{2 \text { max }} \\
\text { (ml.kg-1.min-1) } \\
\text { Potência máxima } \\
\text { (watts) }\end{array}$ & $50.2 \pm 4.5$ \\
Massa corporal $(\mathrm{kg})$ & $73.1 \pm 9.1$ & Mesomorfia & $4.5 \pm 1.2$ & $\begin{array}{c}\text { Potência média } \\
\text { (watts) }\end{array}$ & $483.8 \pm 148.3$ \\
Estatura $(\mathrm{cm})$ & $176.0 \pm 0.1$ & Ectomorfia & $2.0 \pm 0.9$ & IF (watts/s) & $6.7 \pm 3.1$ \\
$\begin{array}{c}\text { Índice de massa } \\
\text { corporal }(\mathrm{kg} / \mathrm{m} 2)\end{array}$ & $23.7 \pm 2.3$ & Somatotipo médio & $\begin{array}{c}\text { Endo } \\
\text { mesomorfo }\end{array}$ & & \\
\hline
\end{tabular}


mente para enfrentar situações que surgem na categoria adulta (Barbieri et al., 2007), como por exemplo, cobranças por resultados e da torcida. Dessa maneira, recomenda-se que os aspetos táticos, técnicos e psicológicos também sejam levados em consideração pela equipe técnica antes da promoção do atleta na categoria adulta. Com isso, alertamos para ainda excessiva preocupação com os fatores antropométricos e fisiológicos, negligenciando outros fatores do mesmo modo importantes para a transição entre as etapas de formação e a idade adulta no futsal.

\section{CONCLUSÕES}

Em conclusão, as categorias Sub-20 e adulta apresentam similar perfil morfológico e desempenho aeróbio e anaeróbio, o que sugere que os atletas da categoria Sub-20 estão aptos, com relação aos aspetos estudados, para atuarem na categoria adulta. No entanto, para futuros estudos seria interessante que aspetos táticos, técnicos e psicológicos fossem analisados para auxiliar na compreensão desta fase de transição e no treinamento dos atletas. Apesar de indicações relevantes no estudo, os resultados devem ser considerados com cuidado, uma vez que apenas duas equipes, uma de cada categoria, foi analisada. Por isso, seria interessante analisar mais atletas de ambas as categorias para fortalecer as conclusões encontradas neste estudo.

\section{Agradecimentos:}

Aos Profs. Tiago Pittoli, José Mario Couto Jr, Marcio Teruo Takayama e o Dr. José Alexandre Curiacos de Almeida Leme pela ajuda nas coletas de dados.

\section{Conflito de Interesses:}

Nada declarado.

\section{Financiamento:}

Nada declarado.
Alvarez, J. C., D’Ottavio, S., Vera, J. G., \& Castagna, C. (2009). Aerobic fitness in futsal players of different competitive level. Journal of Strength and Conditioning Research, 23, 2163-2166.

Amaral, R., \& Garganta, J. (2005). A modelação do jogo em Futsal. Análise sequencial do 1x1 no processo ofensivo. Revista Portuguesa de Ciências do Desporto, 3, 298-310.

Arena, S. S., \& Böhme, M. T. S. (2004). Federações esportivas e organização de competições para jovens. Revista Brasileira de Ciência e Movimento 12, 45-50.

Avelar, A., Santos, K. M., Cyrino, E. S., Carvalho, F. O., Dias, R. M. R., Altimari, L. R., et al. (2008). Perfil antropométrico e de desempenho motor de atletas paranaenses de futsal de elite. Revista Brasileira de Cineantropometria e Desempenho Humano, 10, 76-80.

Barbero-Alvarez, J. C., Soto, V. M., Barbero-Alvarez, V., \& Granda-Vera, J. (2008). Match analysis and heart rate of futsal players during competition. Journal of Sports Science, 26, 63-73.

Barbieri, F. A., Benites, L. C., \& Machado, A. A. (2007). Especialização precoce: algumas implicações relacionadas ao futebol e futsal. In A. A. Machado (Ed.), Especialização esportiva precoce: perspetivas atuais da Psicologia do Esporte (pp. 207-225). Jundiaí: Fontoura.

Barbieri, F. A., \& Gobbi, L. T. B. (2009). Assimetrias laterais no movimento de chute e rendimento no futebol e no futsal. Motricidade, 5(2), 33-47.

Castagna, C., Belardinelli, R., Impellizzeri, F. M., Abt, G. A., Coutts, A. J., \& D’Ottavio, S. (2007). Cardiovascular responses during recreational 5-a-side indoor-soccer. Journal of Science and Medicine Sports, 10, 89-95.

Castagna, C., D’Ottavio, S., Vera, J. G., \& Barbero Alvarez, J. S. (2009). Match demands of professional Futsal: A case study. Journal of Science and Medicine Sports, 12(4), 490-494.

Corrêa, U. C., \& Tani, G. (2006). Esportes coletivos: alguns desafios quando abordados sob uma visão sistêmica. In D. Rose Jr (Ed.), Modalidades esportivas coletivas (pp. 15-23). Rio de Janeiro: Guanabara-Koogan. 
Couto Junior, J. M., Moreno, R. M., Souza, A. F., Prado, M. M., \& Machado, A. A. A influência da torcida na performance de jogadores brasileiros de Futsal: um viés da Psicologia do Esporte. Motriz, 13, 259-265.

Cyrino, E. S., Altimari, L. R., Okano, A. H., \& Coelho, C. F. (2002). Efeitos do treinamento de futsal sobre a composição corporal e o desempenho motor de jovens atletas. Revista Brasileira de Ciência e Movimento, 10, 41-46.

Dias, R. M. R., Carvalho, F. O., Souza, C. F., Avelar, A., Altimari, L. R., \& Cyrino, E. S. (2007). Características antropométricas e de desempenho motor de atletas de futsal em diferentes categorias. Revista Brasileira de Cineantropometria e Desempenho Humano, 9, 297-302.

Dogramaci, S. N., \& Watsford, M. L. (2006). A comparison of two different methods for time-motion analysis in team sports. International Journal of Performance Analysis in Sport, 6, 73-83.

Duarte, R. (2008). Análise da utilização da posse de bola durante o processo ofensivo no futsal - Contributo para a determinação da Eficiência Coletiva. Revista Motricidade, 4, 78-83.

Duarte, M., \& Duarte, C. (2001). Validade do teste aeróbio de corrida de vai-vem de 20 metros. Revista Brasileira de Ciência e Movimento, 9, 7-14.

Garganta, J. (2002). O treino da tática e da técnica nos jogos desportivos à luz do compromisso cognição-ação. In V. Barbanti, A. Amadio, J. Bento, \& A. Marques (Eds.), Esporte e atividade física: interação entre rendimento e saúde (pp. 281-306). São Paulo: Manole.

Heath, B. H., \& Carter, J. E. L. (1967). A modified somatotype method. American Journal of Physical Anthropology, 27, 57-74.

Jackson, A. S., Pollock, M. L., \& Ward, A. (1980). Generalized equations for predicting body density of women. Medicine Science and Sports Exercise, 12, 175-182.

Leal Junior, E. C. P., Souza, F. B., Magini, M., \& Martins, R. A. B. L. (2007). Estudo comparativo do consume de oxigênio e limiar anaeróbio em um teste de esforço progressivo entre atletas profisionais de futebol e futsal. Revista Brasileira de Medicina do Esporte, 12, 323-326.

Léger, L. A., Mercier, D., Gadoury, C. \& Lambertz, J. (1988). The multistage 20 meters shuttle run test for aerobic fitness. Journal of Sports Science, 6, 93-101.

Lima, A. M. J., Silva, D. V. G., \& Souza, A. O. S. (2005). Correlação entre as medidas direta e indireta do $\mathrm{VO}_{2 \max }$ em atletas de futsal. Revista Brasileira de Medicina do Esporte, 11, 164-166.

Machado, J. A., \& Gomes, A. C. (1999). Preparação desportiva no futsal - organização do treinamento na infância e adolescência. Revista Treinamento Desportivo, 4, 55-60.

Queiroga, M. R., Ferreira, A. S., Pereira, G., \& Kokubun, E. (2008). Somatotipo como indicador de desempenho em atletas de futsal feminino. Revista Brasileira de Cineantropometria e Desempenho Humano, 10, 56-61.

Queiroga, M. R., Ferreira, A. S., \& Romanzini, M. (2005). Perfil antropométrico de atletas de futsal feminino de alto nível competitivo conforme a função tática desempenhada no jogo. Revista Brasileira de Cineantropometria $e$ Desempenho Humano, 7, 30-34.

Ré, A. H. N., Teixeira C. P., Massa, M., \& Böhme, M. T. S. (2003). Interferência de características antropométricas e de aptidão física na identificação de talentos no futsal. Revista Brasileira de Ciência e Movimento, 11, 51-56.

Rezer, R., \& Shigunov, V. (2004). Reflexões acerca da prática pedagógica em escolinhas de futebol e futsal a partir da leitura e compreensão de contextos específicos. Revista da Educação Física/ UEM, 15, 43-51.

Ross, W. D., \& Marfell-Jones, M. J. (1982). Kinanthropometry. In J. D. Macdougall, H. A. Wenger \& H. J. Green (Eds.), Physiological testing of the elite athlete (pp. 75-115). New York: Movement Publications.

Santana, W. C., França, V. F., Reis, H. H. B., \& Ribeiro, D. A. (2007). Perfil de iniciação ao futsal de jogadores juvenis paranaenses. Revista Motriz, 13, 181-187.

Siri, W. E. (1961). Body composition from fluid spaces and density. In J. Brozek \& A. Henschel 
70 | F.A. Barbieri, R.A. Barbieri, M.R. Queiroga, W.C. Santana, E. Kokubun

(Eds.), Techniques for measuring body composition (pp. 223-244). Washington: National Academy of Science.

Weineck, J. (2005). Biologia do Esporte (7a ed.). São

Paulo: Manole.

Zacharogiannis, E., Paradisis, G., \& Tziortzis, S.
(2004). An evaluation of tests of anaerobic power and capacity. Medicine Science and Sports Exercise, 36, S116.

(cc) EY-NC

Todo o conteúdo da revista Motricidade está licenciado sob a Creative Commons, exceto quando especificado em contrário e nos conteúdos retirados de outras fontes bibliográficas. 